\title{
Lunar ranging instrument for Chandrayaan-1
}

\author{
J A Kamalakar*, K V S Bhaskar, A S Laxmi Prasad, R Ranjith, K A Lohar, \\ R Venketeswaran and T K Alex \\ Laboratory for Electro-Optics Systems, Indian Space Research Organization (ISRO), Bangalore 560 058, India. \\ *e-mail: kamalakar@leosisro.com
}

Lunar Laser Ranging Instrument (LLRI) proposed for the first Indian lunar mission Chandrayaan-1 is aimed to study the topography of the Moon's surface and its gravitational field by precisely measuring the altitude from a polar orbit around the Moon. Altimetry data close to the poles of the Moon would also be available from the instrument, which was not covered by earlier missions. This instrument supplements the terrain mapping camera and hyper-spectral imager payloads on Chandrayaan-1. The instrument consists of a diode pumped Nd:YAG pulsed laser transmitter having $10 \mathrm{nsec}$ pulse width and a receiver system. The receiver system features $17 \mathrm{~cm}$ diameter Ritchey-Chrétien collecting optics, Si Avalanche Photo Detector (APD), preamplifiers, constant fraction discriminators, time-of-flight measurement unit and spacecraft interface. Altimeter resolution of better than $5 \mathrm{~m}$ is targeted. The received signal strength of LLRI depends on laser pulse backscatter from the Moon's surface. Moon's surface being a poor reflector, the choice of receiver size and its type and the selection of detector play an important role in getting a good signal-to-noise ratio and in turn achieving the target resolution. At the same time, the spacecraft puts a limitation on payload size and weight. This paper discusses the proposed LLRI system for Chandrayaan-1 and signal-to-noise ratio estimation.

\section{Introduction}

Lunar Laser Range Instrument (LLRI) proposed for Chandrayaan-1 is a pulsed laser ranging instrument aimed at enhanced study of the Moon's surface. Advantages of the laser instrumentation are that they can be readily optimized for any given mission scenario. An added advantage is that they can operate from orbiting spacecraft putting comparatively minimal requirements on spacecraft resources in terms of mass, size and electrical power. Being active instruments, laser measurements can be obtained from both the dark and sunlit portions of the Moon, thereby significantly increasing the useful observational coverage. Laser altimetry data from LLRI over the 2-year mission lifetime of Chandrayaan-1 will be used for

- determining the global topography of the Moon,

Keywords. Nd:YAG laser; Si APD; laser altimeter.
- providing an improved model of the lunar gravitational field (Bender et al 1973; Degnan et al 1983),

- interpreting the new topographical and gravitational field data for better understanding of the geophysics of the Moon,

- supplementing the terrain mapping and hyperspectral imager payloads.

\section{Principle of operation}

The principle of LLRI altitude measurement (Degnan et al 1983) is based on the time-of-flight measurement technique in which the range is measured as a direct product of the propagation delay of a laser pulse. By accurately measuring the round trip travel time of the laser pulse from the spacecraft to the Moon's surface, highly accurate spot elevations can be calculated (figure 1). Depending 


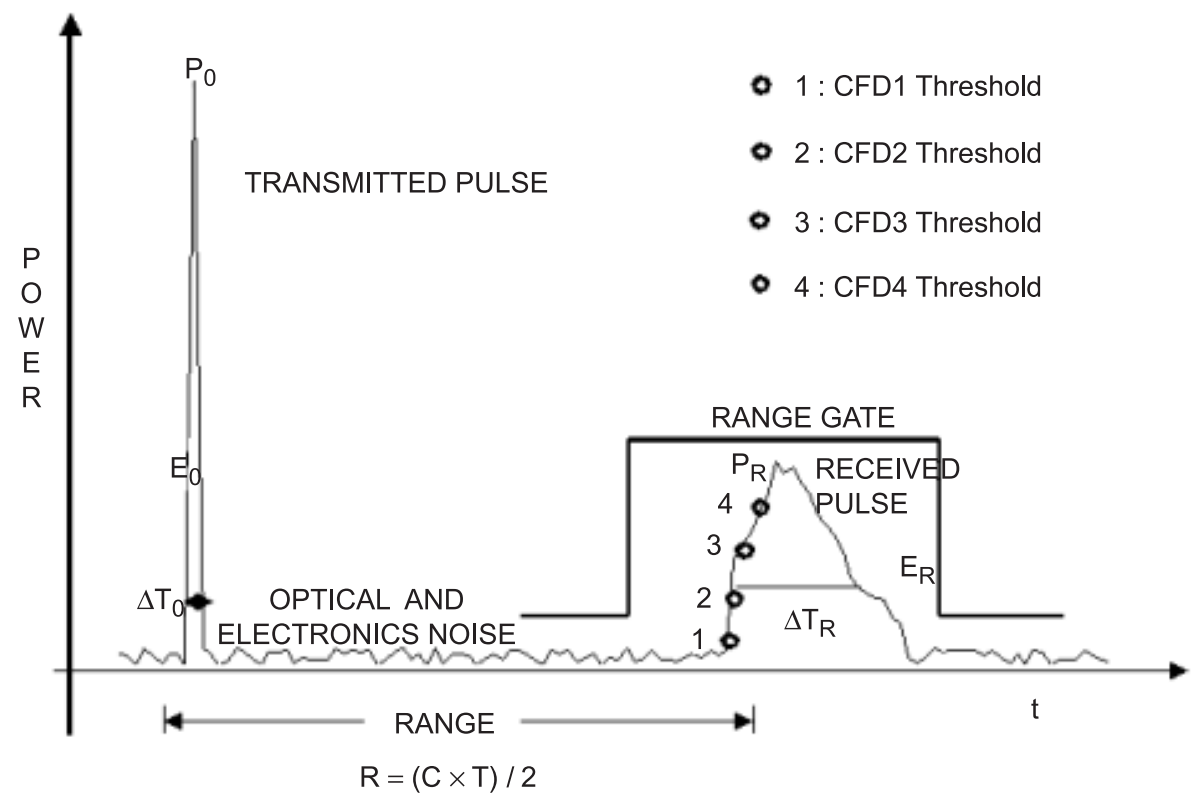

Figure 1. Laser pulse waveform for range measurement.

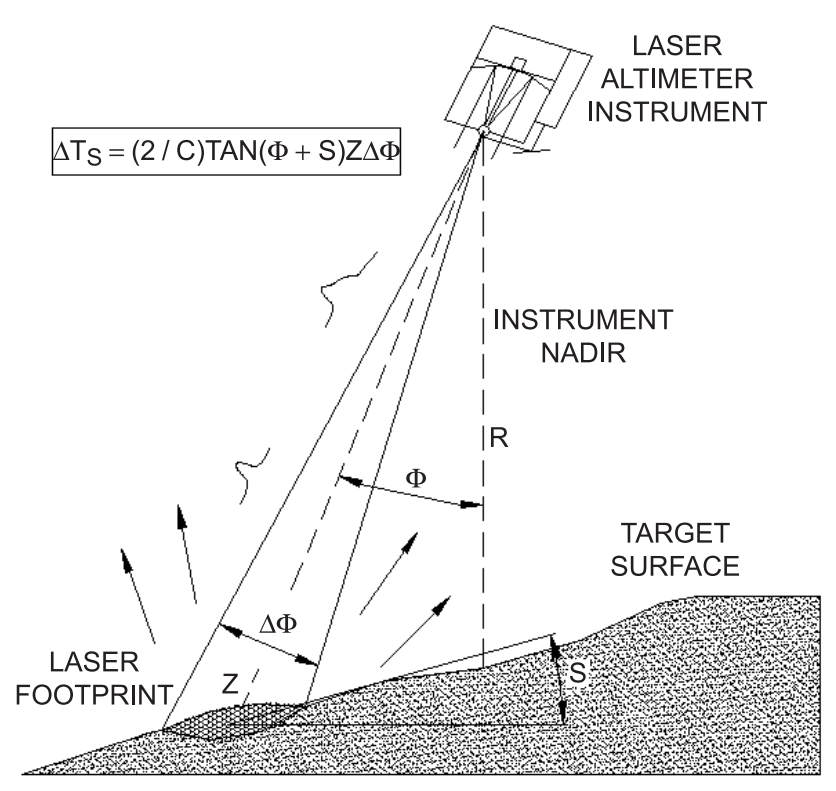

Figure 2. Geometric representation of the laser pulse roundtrip for off-nadir pointing laser altimeter, showing pulse stretching due to terrain slope.

on the altitude and orbital velocity of the spacecraft and laser pulse repetition rate, it is possible to unambiguously obtain range data with high resolution and accuracy. If ' $T$ ' is the total elapsed time, the range ' $R$ ' is given by:

$$
R=\frac{(c \times T)}{2}
$$

where ' $c$ ' is the speed of light in vacuum $\left(2.99792 \times 10^{8} \mathrm{~m} / \mathrm{s}\right)$.

The final accuracy of the range is governed by the uncertainties of the viewing angle (off nadir),
Table 1. Specifications of the lunar laser ranging instrument.

\begin{tabular}{ll}
\hline Laser wavelength & $: 1064 \mathrm{~nm}$ \\
Laser type & $:$ Nd:YAG diode-pumped \\
& Q-switched laser \\
Laser energy & $: 20 \mathrm{~mJ}$ \\
Beam divergence & $: 0.5 \mathrm{mrad}$ (half) \\
Pulse width & $: 10 \mathrm{~ns}$ \\
Pulse repetition rate & $: 10 \mathrm{~Hz}$ \\
Transmitter optics & $: 38 \mathrm{~mm}$ Galilean telescope \\
Receiver optics & $:$ Reflective, $170 \mathrm{~mm}$ \\
Detector & $:$ Avalanche photo detector \\
Vertical resolution & $: 5 \mathrm{~m}$ \\
Footprint on Moon & $: 100 \mathrm{~m}$ \\
Power & $:$ Less than 15 watts \\
Weight & $:$ Less than $10 \mathrm{~kg}$ \\
\hline
\end{tabular}

pointing angle of the laser beam and the time-offlight measurement (figure 2).

\section{LLRI system description}

The system (table 1) needs to be designed in view of the space, mass and power constraints from the spacecraft, at the same time meeting the mission goal of $5 \mathrm{~m}$ accuracy in altitude measurement. Keeping this in view, the transmitter and receiver requirements have been defined. Figure 3 gives the block diagram of LLRI.

\subsection{Laser transmitter}

Laser transmitter power and life play an important role in deciding the size and type of laser. 


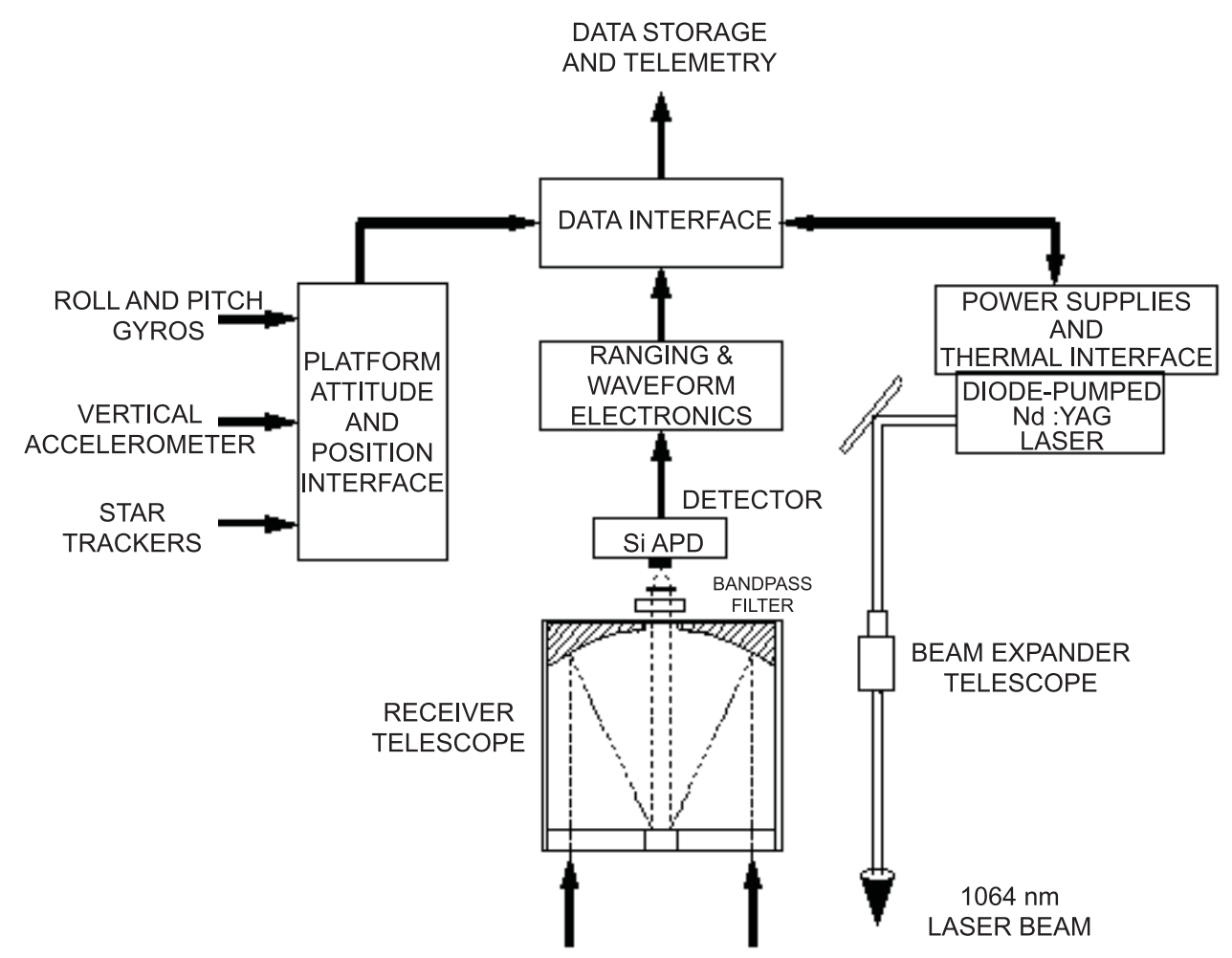

Figure 3. Block schematic diagram of LLRI system.

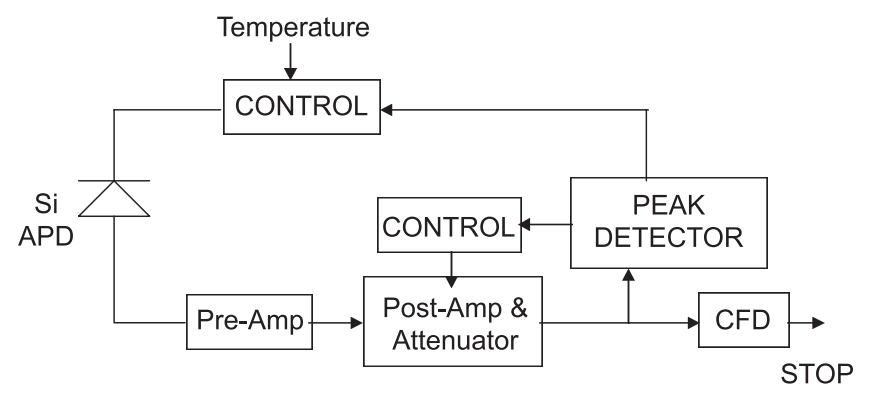

Figure 4. Schematic of receiver electronics front-end.

To keep the transmitter compact and its thermal design simple, a low power transmitter is preferred. However a low power transmitter calls for a bigger receiver aperture to get minimum $S / N$ after the detector. In addition, the received signal from the Moon's surface is very low as its reflectance is typically around $10 \%$ (Nicholas 2004). A tradeoff study was made between transmitter power and receiver aperture size. Finally transmitter power is fixed around a typical value of $20 \mathrm{~mJ}$ considering available sources. Nd:YAG laser operating in pulsed mode at $1064 \mathrm{~nm}$ is considered in view of its flight heritage for such applications. The spacecraft orbits around the moon at a nominal altitude of $100 \mathrm{~km}$. Considering $0.5 \mathrm{mrad}$ half divergence angle for the laser beam, which is achievable comfortably with a minimum of optics, the beam forms a footprint of $100 \mathrm{~m}$ diameter on the Moon's surface. To get contiguous footprints, for the chosen orbit, the laser has to operate at $10 \mathrm{~Hz}$ repetition frequencies. For a 2-year mission, it comes to be $64 \times 10^{7}$ pulses. Since this type of life requirement cannot be met by flash lamp pumped laser, diode pumped solid-state laser is preferred for transmitter. In addition, such a system is compact and requires low power. Further Q-switched laser is preferred to get narrow pulses, which also improves the accuracy of measurement.

\subsection{Receiver telescope}

The purpose of the receiver telescope is to collect the lunar reflected infrared altimetry signal and focus it on to an avalanche photodiode. RitcheyChrétien telescope configuration was considered for LLRI as this type of configuration leads to coma free designs, low light losses and contains no refractive elements. In addition, this type of configuration leads to good off-axis performance, the precise combination of surface form is chosen to preserve the high on-axis resolution while giving a wider useful field-of-view. With a transmitter energy being $20 \mathrm{~mJ}$, to get optimum $S / N$ after taking into account the worse case reflection and surface slope, the receiver aperture size is fixed at $170 \mathrm{~mm}$.

\subsection{Receiver electronics}

The front end of LLRI must process signals (Paulus 1985) in the nano-second range. The 


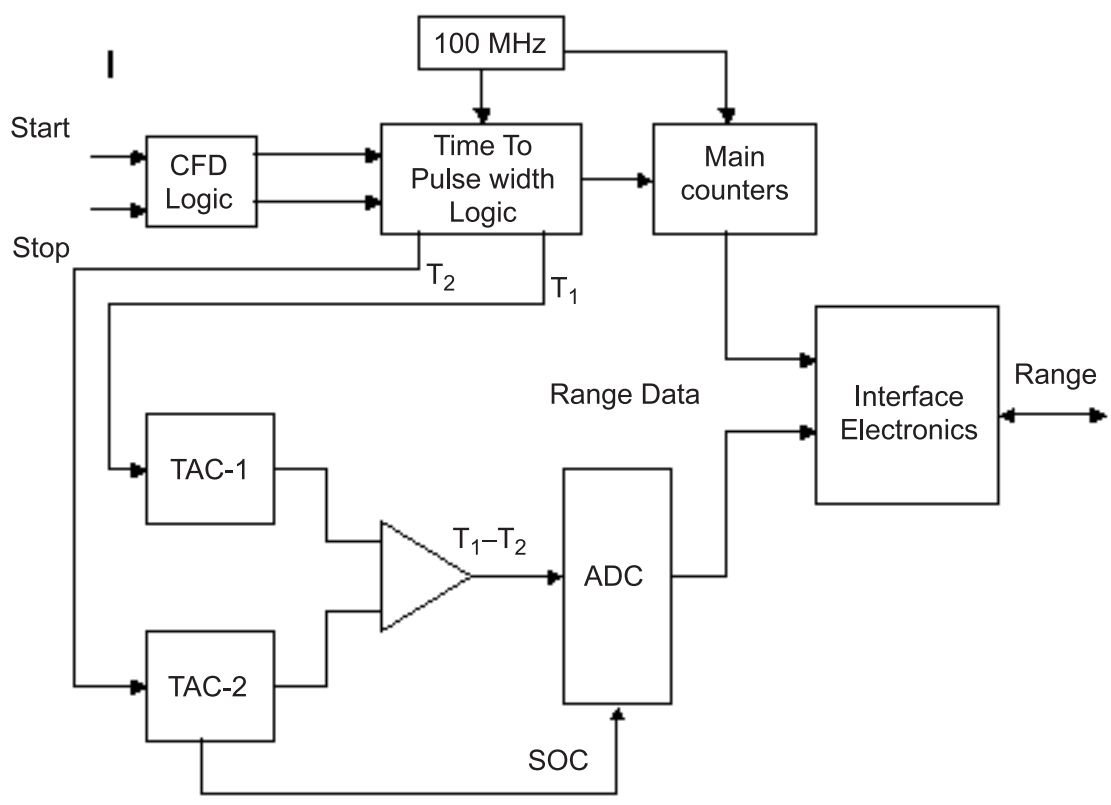

Figure 5. Block schematic of receiver range electronics.

Table 2. Laser energy: $20 \mathrm{~mJ}$, receiver dia: $170 \mathrm{~mm}$, pulse width: $10 \mathrm{~ns}$, range: $100 \mathrm{~km}$, detector responsivity: $200 \mathrm{kV} / \mathrm{W}$.

\begin{tabular}{|c|c|c|}
\hline $\begin{array}{l}\text { Off-nadir angle } \\
\text { in degree }\end{array}$ & $\begin{array}{l}\text { Moon's surface slope } \\
\text { in degree }\end{array}$ & $S / N$ \\
\hline \multirow{4}{*}{0} & $(0$ & 44.1 \\
\hline & $\{0.1$ & 42.8 \\
\hline & 1 & 35.6 \\
\hline & 5 & 20.2 \\
\hline \multirow{4}{*}{0.05} & $(0$ & 43.6 \\
\hline & 0.1 & 42.4 \\
\hline & \{ & 35.4 \\
\hline & 5 & 20.2 \\
\hline
\end{tabular}

quality of these signals is very important. The block schematic of the receiver electronics is shown in figures 4 and 5 . The main sub-elements of the receiver electronics are the detector, constant fraction discriminators (CFD) and time digitizer unit (TDU).

\section{Signal-to-noise $(S / N)$ considerations}

The resolution of LLRI measurement is determined by the $S / N$ of detection. Analyses for lunar ranging using LLRI indicate that about 1800 photoelectrons will be generated for every $20 \mathrm{~mJ}$ of transmitted energy. The beam divergence of $0.5 \mathrm{mrad}$ (half divergence) and a Galilean beam expander guarantee that the $100 \mathrm{~m}$ lunar footprints can be realized with adequate energy being returned to the receiver system. The pulse repetition rate of $10 \mathrm{~Hz}$ determines the number of observations from the lunar surface.

\subsection{Signal}

For lunar ranging operation, the received power can be approximated by using the equation

$$
P_{s}=\frac{P_{T} G_{T}}{4 \pi R^{2}} \times \frac{\sigma}{4 \pi R^{2}} \times \frac{\pi D^{2}}{4} \times \eta_{\mathrm{atm}} \eta_{\mathrm{sys}},
$$

where, $P_{s}$ is the received signal power (watts), $P_{T}$ is the transmitted power (watts), $G_{T}$ is the transmitter gain given by

$$
G_{T}=\frac{16}{\theta_{T}^{2}}
$$

where, $\theta_{T}=K_{a} \lambda / D$, is the transmitter beam divergence. $K_{a}=$ aperture illumination constant, $\lambda$ is the wavelength, $D$ is the aperture diameter, $\sigma$ is the effective target cross-section and is defined as:

$$
\sigma=\frac{4 \pi}{\Omega} \times \rho_{T} \times d A
$$

where, $\Omega$ is the scattering solid angle of target (steradian), $\rho_{T}$ is the target reflectivity, $d A$ is the target area on which the beam is falling, $R$ is the system range to target, $D$ is the aperture diameter, $\eta_{\text {atm }}$ is the atmosphere transmission factor, $\eta_{\text {sys }}$ is the system transmission factor $\left(=T_{r} T_{o}\right), T_{r}$ is the transmission of transmitter, and $T_{o}$ is the transmission of receiver optics.

Considering a diffused lambertian target $(\Omega=\pi)$

$$
\sigma=4 \rho_{T} d A
$$




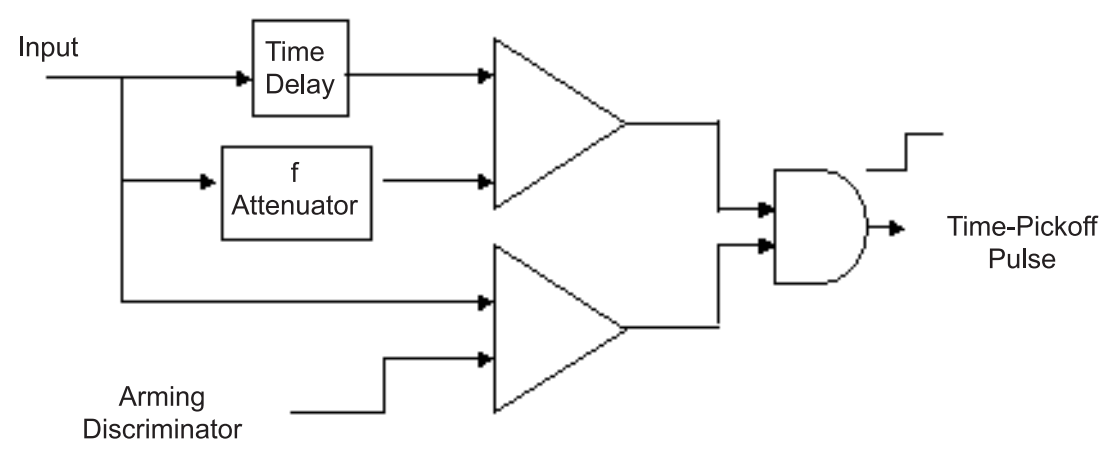

Figure 6. CFD block schematic.

For target normal to beam,

$$
d A=\frac{\pi R^{2} \theta_{T}^{2}}{4}
$$

and, $\sigma=\sigma_{\mathrm{EXT}}=\pi \times \rho_{T} \times R^{2} \times \theta_{T}^{2}$.

Based on the above equations, the received signal power at the detector is calculated as:

$$
P_{s}=\frac{P_{T} \rho_{T} A_{T} T_{r} T_{o}}{\pi R^{2}} .
$$

\subsection{Noise}

The main source of noise is typically the shot noise, detector dark current and background signal from terrain. The detector mean square noise current is given by

$$
\left\langle i_{n}^{2}\right\rangle=2 q\left[I_{D S}+\left(I_{D B}+P_{O} R_{O}\right) M^{2} F\right] B W_{N}
$$

where $q$ is the charge of the electron, $I_{D S}$ is the dark current with avalanche gain, $I_{D B}$ is the bulk current without avalanche gain, $P_{O}$ is the total flux incident on the detector (watts), $R_{O}$ is the unity gain responsivity $(A / W), M$ is the avalanche gain (multiplication factor), $F$ is the excess noise factor, and $B W_{N}$ is the noise equivalent bandwidth.

The electrical signal power $i_{s}^{2}$ is given by

$$
i_{s}^{2}=\left(P_{s} R_{O} M\right)^{2} \quad\left[\text { ampere }^{2}\right]
$$

where, $P_{S}$ is peak signal power (watts), obtained from equation (7).

The electrical noise power $i_{n}^{2}$ includes shot noise plus amplifier noise:

$$
i_{n}^{2}=\left\{2 q\left[I_{D S}+\left(I_{D B}+P_{O} R_{O}\right) M^{2} F\right]+i_{N A}^{2}\right\} B W_{N}
$$

where, $i_{N A}$ is the amplifier input r.m.s noise current spectral density in $\left[\right.$ ampere $\left./(\mathrm{Hz})^{1 / 2}\right]$. The signalto-noise ratio is thus given by:

$$
\begin{aligned}
\frac{S}{N} & =\frac{i_{s}^{2}}{i_{n}^{2}} \\
& =\frac{\left(P_{s} R_{O} M\right)^{2}}{\left\{2 q\left[I_{D S}+\left(I_{D B}+P_{O} R_{O}\right) M^{2} F\right]+i_{N A}^{2}\right\} B W_{N}} .
\end{aligned}
$$

\subsection{Range resolution}

The finite $S / N$ can cause a timing uncertainty in the range measurement. The r.m.s range error $\Delta R$ is given by:

$$
\Delta R=\frac{c \Delta t_{r}}{\left[2 \times(n \times S / N)^{1 / 2}\right]},
$$

where, $\Delta t_{r}$ is the laser pulse width, $n$ is the number of laser shots per measurement (here $n=1$ ) and $c$ is the velocity of light in vacuum. Another source of ranging uncertainty results from pulse spreading. For a laser beam width $\Delta \phi$, an angular offset $\phi$ from nadir and a surface slope $S$, the pulse spread $\Delta T_{S}$ beyond the nominal laser pulse width can be determined by:

$$
\Delta T_{S}=(2 / c) \tan (\phi+S) R \Delta \phi
$$

where, $R$ is the range to lunar surface.

\subsection{Detector and $S / N$ estimation}

The input signal to the receiver is a small fraction of the reflected laser pulse from the lunar surface collected by the receiver telescope. The two alternatives for the detectors are PIN photodiodes and avalanche photodiode (APD). Considering the factors like size, sensitivity, speed of response and ease of use, Si APD is chosen for LLRI. An added advantage of using Si APD is that the internal gain mechanism of APD improves the noise equivalent power (NEP) of the receiver as compared to PIN diodes.

Table 2 provides the variation of $S / N$ with off nadir angle and slope of Moon's surface for the chosen parameters for transmitter and receiver. Even 


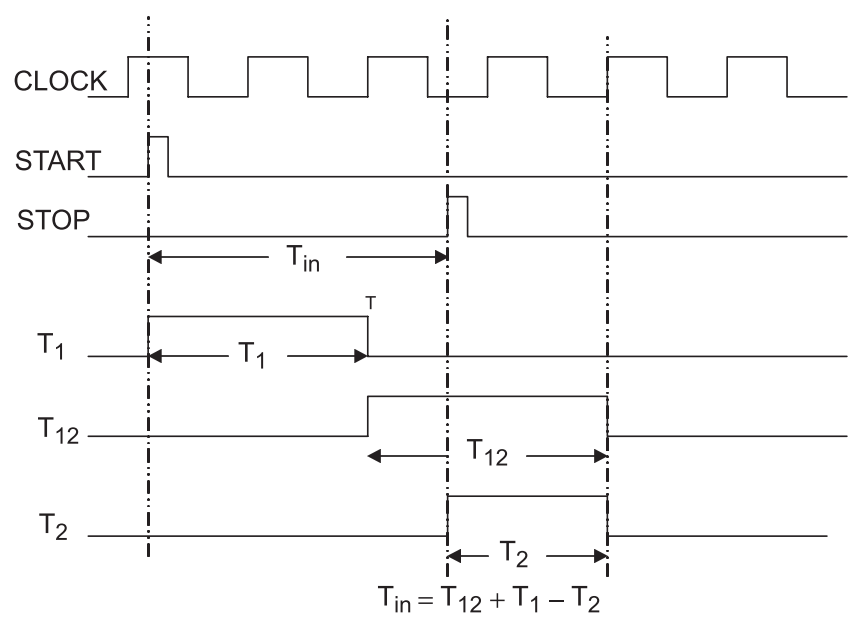

Figure 7. Block diagram and operating principle of time digitizer.

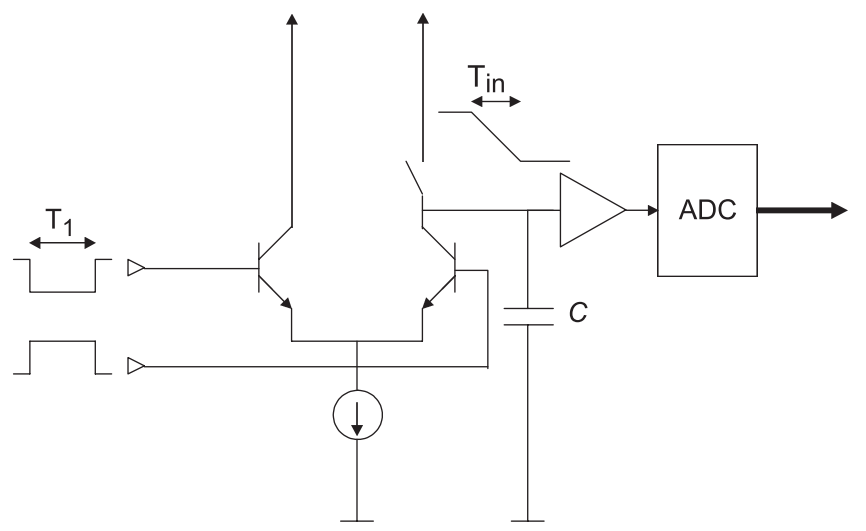

Figure 8. Analog interpolator principle of operation.

with worst case $S / N$ of 20 , range accuracy of less than $5 \mathrm{~m}$ can be comfortably achieved.

\section{Description of receiver elements}

\subsection{Constant Fraction Discriminator (CFD)}

The amplitude of the reflected received signal varies over a wide range depending on the range and reflectivity of the lunar terrain. The timing event should not change when the signal level varies as it directly affects the measurement results (Sperr and Maier 1970). Receiver front-end electronics should produce accurately timed logic pulses from the optical input pulses of varying amplitudes. The timing event can be generated by leading edge discrimination or constant fraction discrimination (CFD) techniques. As leading edge discrimination technique leads to walk errors, CFD technique is chosen for LLRI. In CFD (figure 6), the event timing is generated by comparing the attenuated signal and the direct signal so that their crossing point defines a constant fraction of the pulse. By this method, the timing point is made insensitive to variation in the amplitude of the signal.

\subsection{Time digitizer unit (TDU)}

The TDU forms the heart of the LLRI processing electronics. The outputs of the CFD are fed to the TDU. The TDU measures the time interval between the 'start' and 'stop' pulses generated by the CFDs. This timing interval between the start and stop signals can be measured by analog or digital methods. The analog method is based on the charging or discharging of a known capacitor by a constant current, the voltage drop across the capacitor being linearly dependent on the discharging time. The single shot precision and integral non-linearity of the analog method depends on the measurement range. The analog method has good single-shot precision and is suitable for short-range measurements, but suffers from poor stability. The digital method is based on the synchronous counting of the clock pulse from a stable reference oscillator during the measured time interval. Though the measurement is inherently linear over a wide measurement range, this method suffers from poor resolution, which is \pm 1 clock cycle. For LLRI having wide measurement range, high precision and accuracy, the time interval measurement is done utilizing the digital methods for achieving improved single-shot precision and high linearity with high measurement speed and the one-clock resolution.

\subsubsection{TDU operating principle}

Figure 7 illustrates the time interval measurement principle. The measured time interval $T$ between the start and stop pulses can be divided into three parts, $T_{12}, T_{1}$ and $T_{2}$ as shown in figure 7 in such a way that

$$
T=T_{12}+T_{1}-T_{2} .
$$

The main part $T_{12}$ is synchronous with respect to the system clock and can therefore be digitized by counting clock pulses. With a counter and a $100 \mathrm{MHz}$ clock, a single-shot measurement resolution of $10 \mathrm{~ns}$ can be achieved. Since the measurement is asynchronous with respect to the system clock, averaging can be used to improve resolution. However, the measurement time increases, so to improve the single-shot resolution, the nonsynchronous parts $T_{1}$ and $T_{2}$ are digitized separately with interpolators. Thus, the measurement range is set by the number of bits in the counter and limited only by the stability of the system clock.

The principle of the interpolator (Kalisz and Mpawloski 1987) is based on the time-to-voltage 
conversion. As shown in figure 8, a capacitor $(C)$ is discharged with a constant current $(I)$ during the input time interval $\left(T_{\text {in }}\right)$. The resulting voltage change $\Delta V=(I / C) \times T_{\text {in }}$ is then digitized with an $N$-bit $A / D$ converter, so that the LSB width of the time digitizer is equal to $T_{\mathrm{CLK}} / 2^{N}$. The gain of the time-to-voltage conversion depends on the absolute values of the capacitance and the current, so the interpolators need to be calibrated. From these results the gains of both the interpolators can be calculated and these values can be used during measurement.

The time interval data are time-tagged with respect to the spacecraft time frame. The time stamping is for use with the spacecraft orbit and attitude information for determining the alongtrack location of each laser footprint on the lunar surface.

\section{Conclusion}

Chandrayaan-1 will be the first Indian space mission to the moon. The payloads, i.e., terrain mapping camera, hyper-spectral imager, X-ray and lunar laser ranging instrument have been chosen to meet the scientific objectives of providing high resolution remote sensing of the Moon. The lunar laser ranging instrument which is developed by the Laboratory for Electro-Optics Systems, will provide high-resolution altimetry for supplementing the tracking and elevation model from the terrain mapping camera and will further be used for improving the gravity model. In addition, the polar orbit lunar mission will serve in enhancing the altimetry database near the lunar poles.

\section{References}

Bender Currie P L, Dicke D G, Eckhardt R H, Faller D H and Wilson E C 1973 The lunar laser ranging experiment; Science 182229.

Degnan J J, Kahn W D and Englar T S 1983 Centimeter precision airborne laser ranging systems; J. Survey. Engineer. 109(2) 99-115.

Kalisz J and Mpawloski R Pelka 1987 A multiple interpolation method for fast and precise time digitizing; IEEE Transactions on Instrumentation and Measurements 35 163-169.

Nicholas M Short 2004 Pre-Apollo Exploration of the moon GSFC (Goddard Space flight centre), NASA.

Paulus T J 1985 Timing electronic and fast timing methods with scintillation detectors; IEEE Transactions on Nuclear Science 3 1242-1249.

Sperr P and Maier M R 1970 On the construction of a fast constant fraction trigger with integrated circuits and application to various photomultiplier tubes; Nuclear Instruments and Methods 87 13-18. 